\title{
HANDLEBODY COMPLEMENTS IN THE 3-SPHERE: A REMARK ON A THEOREM OF FOX
}

\author{
MARTIN SCHARLEMANN
}

(Communicated by James E. West)

\begin{abstract}
Let $W$ be a compact 3-dimensional submanifold of $S^{3}$, and $C$ be a collection of disjoint simple closed curves on $\partial W$. We give necessary and sufficient conditions (one extrinsic, one intrinsic) for $W$ to have an imbedding in $S^{3}$ so that $S^{3}-W$ is a union of handlebodies, and $C$ contains a complete collection of meridia for these handlebodies.
\end{abstract}

A theorem of Fox [F] says that any connected compact 3-dimensional submanifold $W$ of $S^{3}$ is homeomorphic to the complement of a union of handlebodies in $S^{3}$. Call a collection $C$ of simple closed curves in $\partial W$ protomeridinal if there is some embedding of $W$ in $S^{3}$ so that the complement of $W$ is a union of handlebodies and $C$ contains a complete collection of meridia for this handlebody. It is natural to ask whether a given collection $C$ of simple closed curves in $\partial W$ is proto-meridinal. This is a difficult question to answer. For example, if $\partial W$ is a torus, either $W$ is a solid torus or only one curve in $\partial W$ is proto-meridinal. The proof is essentially the celebrated solution by Gordon and Luecke [GL] of the knot complement conjecture.

For genus $(\partial W)>1$ the problem seems intractable. But it does make sense to ask the following question: What conditions on the given embedding of $W$ in $S^{3}$ (called extrinsic conditions) and what conditions on the pair $(W, C)$ itself (called intrinsic conditions) suffice to guarantee that $C$ is proto-meridinal? The general goal is to discover intrinsic conditions that allow extrinsic conditions to be weakened. Here it is shown that a simple intrinsic condition allows one to reduce the extrinsic requirements to a simple condition on $C$ regarded as a link in $S^{3}$.

Let $C$ be a collection of disjoint simple closed curves in $\partial W \subset W \subset S^{3}$. The normal direction to $\partial W$ in $S^{3}$ determines (up to orientation) a framing of the normal bundle $\eta(C)$ of $C$ in $S^{3}$. Suppose $C$ is the unlink in $S^{3}$ and $\Delta$ is a collection of disjoint disks that $C$ bounds. Then the normal of $C$ into $\Delta$ also determines (up to orientation) a framing on $\eta(C)$. If the framings coincide (up to orientation) then we say that $C$ is a framed unlink.

A collection of disjoint simple closed curves in the boundary of a handlebody

Received by the editors January 3, 1991.

1991 Mathematics Subject Classification. Primary 57M25, 57M40.

The author was supported in part by a National Science Foundation grant.

(C) 1992 American Mathematical Society $0002-9939 / 92 \$ 1.00+\$ .25$ per page 
is a complete collection of meridia if it bounds a set of disjoint disks whose complementary closure is the 3-ball.

Theorem. Suppose $W$ is a connected compact 3-dimensional submanifold of $S^{3}$ and $C$ is a family of disjoint simple closed curves in $\partial W$ that is a framed unlink in $S^{3}$. Then $C$ is proto-meridinal if and only if $H_{2}(W, \partial W-C) \rightarrow$ $\mathrm{H}_{2}(W, \partial W)$ is trivial.

Proof. The condition on $\mathrm{H}_{2}$ can be stated geometrically: Any properly embedded surface in $W$ whose boundary is disjoint from $C$ must be separating.

One direction is easy. Suppose $S^{3}-W$ is a union $H$ of handlebodies and $C$ contains a complete set of meridia for $H$. Then any simple closed curve in $\partial W-C$ bounds a disk in $H$. Hence any properly embedded surface $S$ in $W$ whose boundary lies in $\partial W-C$ can be capped off to give a closed surface in $S^{3}$, which must be separating. Then $S$ is separating in $W$.

For the other direction, we first find a compressing disk $E$ for $\partial W$ in $S^{3}-W$ such that $\partial E$ is disjoint from $C$. Recall that $C$ is the framed unlink. Let $\Delta$ denote a disjoint collection of disks bounded by $C$ in $S^{3}$, chosen to minimize the number of components of intersection with $\partial W$. Since the framing of $\eta(C)$, given by the normal into $\Delta$ coincides with the framing given by the normal to $\partial W$ in $S^{3}, \Delta$ can be put in general position with respect to $\partial W$ so that $\Delta \cap \partial W$ consists of a set of simple closed curves in $\Delta$, possibly just $C$. Choose $\Delta$ so as to minimize the number of components of $\Delta \cap \partial W$. Then an innermost circle of intersection of $\partial W$ with $\Delta$ (or a component of $\partial \Delta$ if $\Delta \cap \partial W=\partial \Delta$ ) is an essential circle in $\partial W$ that is disjoint from (or can be isotoped off of) $\partial \Delta=C$ and bounds a disk $E$ in $S^{3}-\partial W$.

We now proceed by induction on $\beta_{1}-\beta_{0}$, where $\beta_{i}$ is the $i$ th betti number of the union of all nonspherical components of $\partial W$. If $\beta_{1}-\beta_{0}=0$, then $\partial W$ consists of spheres and there is nothing to prove. The hypothesis is that $\mathrm{H}_{2}(W, \partial W-C) \rightarrow H_{2}(W, \partial W)$ is trivial. With no loss we may assume all curves of $C$ are essential in $\partial W$. Consider the following possibilities for $E$ :

Case 1. $E$ lies in $W$. Since $H_{2}(W, \partial W-C) \rightarrow H_{2}(W, \partial W)$ is trivial, $E$ is separating, and so decomposes $W$ into the boundary connected sum of two manifolds $W_{1}$ and $W_{2}$, each with boundary of lower genus than that of $W$. By inductive assumption, each $W_{i}$ can be embedded in the 3-sphere so that $S^{3}-W_{i}$ is a union of handlebodies $H_{i}$ in which some subset $C_{i}$ of $C \cap \partial W_{i}$ bounds a complete collection of meridia. Then the connected sum of these spheres along 3-balls bisected by the equatorial disk $E \subset \partial W_{i}$ gives an embedding of $W$ in $S^{3}$ whose complement is the boundary sum of $H_{1}$ and $H_{2}$, hence a union of handlebodies, in which $C_{1} \cup C_{2} \subset C$, is a complete collection of meridia.

Case 2. $E$ lies in $S^{3}-W$. Let $W^{\prime}$ be the manifold obtained from $W$ by attaching a 2-handle $\eta(E)$ along $E$. Let $C^{\prime}$ be the subcollection of $C$ that remains essential in $\partial W^{\prime}$. Suppose there were a nonseparating surface $S^{\prime}$ in $W^{\prime}$ with $\partial S^{\prime} \subset \partial W^{\prime}-C^{\prime}$. Any element of $C-C^{\prime}$ is inessential in $\partial W^{\prime}$ so we may isotope $\partial S^{\prime}$ to be disjoint from $C$. By general position we may take $S^{\prime} \cap \eta(E)$ to be disks parallel to $E$. Then $S=S^{\prime} \cap W$ would be a nonseparating surface in $W$ with $\partial S \subset \partial W-C$. We conclude that such a surface $S^{\prime}$ cannot exist; i.e., $H_{2}\left(W^{\prime}, \partial W^{\prime}-C^{\prime}\right) \rightarrow H_{2}\left(W^{\prime}, \partial W^{\prime}\right)$ is trivial. By induction, there is an embedding of $W^{\prime}$ in $S^{3}$ so that $S^{3}-W^{\prime}$ is a union of handlebodies $H^{\prime}$ in which $C^{\prime}$ bounds a complete collection of meridia. Now remove the 2-handle 
to regain $W$. Its complement $H$ is obtained by attaching a 1-handle to $H^{\prime}$, so $H$ is also a union of handlebodies.

Subcase (a). $E$ is separating. Then $C^{\prime}$ also contains a complete collection of meridia for $S^{3}-W$.

If $E$ is nonseparating, the sides of the 2-handle $E_{ \pm}$lie on the same component $T^{\prime}$ of $\partial W^{\prime}$. Let $T$ be the corresponding component of $\partial W$, containing $\partial E$.

Subcase (b). $E_{ \pm}$lie in different components of $T^{\prime}-C$. Then there is a curve $c^{\prime} \in C$ that separates $T^{\prime}$ so that $E_{+}$and $E_{-}$lie in different components of $T^{\prime}-c^{\prime}$. Since $C^{\prime} \subset C$ contains a complete collection of meridia $C^{\prime \prime}$ of $H^{\prime}$, it follows that $c^{\prime}$ bounds a separating disk $D$ in $H^{\prime}$. After sliding the entire contents (handles and curves) of one component of $H^{\prime}-D$ over the 1-handle dual to $E, D$ becomes the cocore of that 1-handle and $C^{\prime \prime} \cup c^{\prime} \subset C$ becomes a complete collection of meridia curves for $H$.

Subcase (c). $E_{ \pm}$lie in the same component of $T^{\prime}-C$. Then there is a circle $d$ in $T-C$ intersecting $\partial E$ precisely once. If $T$ is not a torus, then banding together $E_{ \pm}$along $d-E$ gives an essential separating disk in $S^{3}-W$ with boundary in $T-C$, i.e., Subcase (a). If $T$ is a torus, then $\partial E \cup d$ is a spine of $T$, so $T$ is disjoint from $C$. Consider $Q=\partial W-T$. The image of $H_{1}(Q-C)$ in $H_{1}(Q)$ has rank at least genus $(Q)=\operatorname{genus}(\partial W)-1$. Hence the image of $H_{1}(\partial W-C)$ in $H_{1}(\partial W)$ has rank at least genus $(Q)+\beta_{1}(T)=$ genus $(\partial W)+1$. But it is a well-known consequence of Poincare duality that the image of $H_{1}(\partial W)$ in $H_{1}(W)$ has rank $=\operatorname{genus}(\partial W)$. Hence there is an $\alpha$ in $H_{1}(\partial W-C)$ that maps nontrivially to $H_{1}(\partial W)$ but trivially to $H_{1}(W)$. Then an element in $H_{2}(W, \partial W-C)$ mapping to $\alpha$, cannot map trivially to $H_{2}(W, \partial W)$. This contradicts the hypothesis that $H_{2}(W, \partial W-C) \rightarrow$ $\mathrm{H}_{2}(W, \partial W)$ is trivial.

Remark. The condition on framings is necessary: Let $T$ be the standard unknotted torus in $S^{3}$, separating $S^{3}$ into two solid tori, one with meridian $\mu$, and the other with meridian $\lambda$. Let $W=T \times I$ be a collar so that $\mu \times\{0\}$ and $\lambda \times\{1\}$ are meridia for the solid tori $S^{3}-W$. Let $c_{1}=\mu \times\{0\}$ and $c_{2}$ be the curve in $T \times\{1\}$ homologous to $\mu+p \lambda,|p|>1$. Then $c_{1} \cup c_{2}$ is the unlink in $S^{3}$ and $H_{2}(W, \partial W-C)=0$. But since in $T, c_{1} \cdot c_{2}=p \neq \pm 1$, there is no embedding of $W$ in $S^{3}$ for which $c_{1}$ and $c_{2}$ are meridia of distinct complementary solid tori.

To see the difficulty, note that any disk that $c_{2}$ bounds in $W$ will have a transverse intersection arc with $\partial W$. This arc is a consequence of the mismatch of framings and immediately prevents the construction of $E$ in the proof of the theorem.

\section{REFERENCES}

[F] R. Fox, On imbeddings of polyhedra in 3-space, Ann. of Math. (2) 49 (1948), 462-470.

[GL] C. Gordon and J. Luecke, Knots are determined by their complements, J. Amer. Math. Soc. 2 (1989), 371-415.

Department of Mathematics, University of California, Santa Barbara, California 